\title{
An Action-Related Theory of Causality Donald Gillies
}

\begin{abstract}
The paper begins with a discussion of Russell's view that the notion of cause is unnecessary for science, and can therefore be eliminated. It is argued that this is true for theoretical physics, but untrue for medicine where the notion of cause plays a central role. Medical theories are closely connected with practical action (attempts to cure and prevent disease), whereas theoretical physics is more remote from applications. This suggests the view that causal laws are appropriate in a context where there is a close connection to action. This leads to a development of an action-related theory of causality which is similar to the agency theory of Menzies and Price, but differs from it in a number of respects, one of which is the following. Menzies and Price connect 'A causes B' with an action to produce $\mathrm{B}$ by instantiating $\mathrm{A}$, but, particularly in the case of medicine, the law can also be linked to the action of trying to avoid B by ensuring that $\mathrm{A}$ is not instantiated. The action-related theory has in common with agency theory of Menzies and Price, the ability to explain causal asymmetry in a simple fashion, but the introduction of avoidance actions together with some ideas taken form Russell enable some of the objections to agency accounts of causality to be met.

1 Introduction

2 Russell on Causality

3 Preliminary Exposition of the Action-Related Theory

4 Differences between the Action-Related Theory, and the Agency Theory of Menzies and Price

5 Explanation of Causal Asymmetry

6 Objections to the Action-Related Theory

7 Extension of the Theory to the Indeterminate Case
\end{abstract}

\section{Introduction}

The aim of this paper is to present an action-related theory of causality. This theory is a variant of the agency theory of causality which has been expounded by Peter Menzies and Huw Price in an important series of papers (Price [1991], [1992] and Menzies and Price [1993]). Accordingly when developing the action-related theory, I will compare it to the agency theory of Menzies and Price, pointing out similarities and differences. I have invented a new name to capture the differences between the two theories, but it should be stressed that there are important agreements as well, particularly as regards the key issue of explaining the asymmetry of the causal relation. 
The general approach of relating causality to human action or agency has in fact quite a long history. Menzies and Price mention some earlier writers ([1993], p. 187, Footnote 1), and in fact the basic idea is to be found in the following famous quotation from Bacon ([1620], III, p. 259):

Human knowledge and human power meet in one, for where the cause is not known the effect cannot be produced. Nature to be commanded must be obeyed; and that which in contemplation is as the cause is in operation as the rule.

A connection between cause and action is also mentioned by Kant ([1781/7], A204/B249): 'Causality leads to the concept of action ...' However this remark is not followed up in any detail, and Kant's overall position on causality obviously cannot be described as an agency or action-related theory.

It would be interesting to trace this earlier history in more detail, but, as I want to concentrate in this paper on the current issues, I will confine myself to discussing briefly one famous paper on causality, even though this does not present, or even mention, an action or agency approach. The paper in question is Russell's [1913] 'On the Notion of Cause'. This paper has a particular interest for me since I believed its conclusions for many years, and even though I now reject Russell's overall position, I still think that some of his ideas are valid, and will attempt to incorporate these ideas in the view to be presented in what follows.

\section{Russell on Causality}

Russell begins his paper in a dramatic fashion by claiming the word 'cause' should be altogether banished from philosophy ([1913], p. 173):

In the following paper I wish, first, to maintain that the word 'cause' is so inextricably bound up with misleading associations as to make its complete extrusion from the philosophical vocabulary desirable, ...

Russell's principal reason for this recommendation is that the word 'cause' has already disappeared from the advanced sciences. As he says ([1913], p. 173):

All philosophers, of every school, imagine that causation is one of the fundamental axioms or postulates of science, yet, oddly enough, in advanced sciences such as gravitational astronomy, the word 'cause' never occurs.

Russell, writing just before the First World War, concludes that causality, like the British monarchy, is something no longer appropriate in the modern age. With his customary wit, he puts the point as follows ([1913], p. 173):

The law of causality, I believe, like much that passes muster among philosophers, is a relic of a bygone age, surviving, like the monarchy, only because it is erroneously supposed to do no harm.

In these quotations Russell has perhaps rather exaggerated his own position in the interest of some striking turns of phrase. If we read on, we discover that he does after all 
allow a weak notion of causality. However he claims that this notion of causality is useful only in everyday life and the infancy of science. His main thesis, therefore, is that the concept of cause disappears from science as it advances.

Regarding the weak notion of causality which he in fact allows, Russell gives an analysis similar to Hume's 'constant conjunction' account, except that Russell claims that the sequences are of frequent rather than absolutely constant conjunction, and that they yield no more than probability ([1913], p. 185):

... the sequence, in any hitherto unobserved instance, is no more than probable, whereas the relation of cause and effect was supposed to be necessary. ... Thus in our present sense, A may be the cause of B even if there actually are cases where B does not follow A. Striking a match will be the cause of its igniting, in spite of the fact that some matches are damp and fail to ignite.

Thus causal laws for Russell are laws of probable sequence. He points out one curious consequence of this neo-empiricist or neo-Humean account, namely that it turns out to be correct to say that night causes day ([1913], p. 185). Intuitively however the statement that night causes day does not seem to be true.

In terms of the analysis of causal laws as laws of probable sequence, Russell is in a position to state his main thesis which he does as follows ([1913], p. 186):

... such laws of probable sequence, though useful in daily life and in the infancy of science, tend to be displaced by quite different laws as soon as a science is successful. The law of gravitation will illustrate what occurs in any advanced science. In the motions of mutually gravitating bodies, there is nothing that can be called a cause and nothing than can be called an effect; there is merely a formula. Certain differential equations can be found, which hold at every instant for every particle of the system, and which, given the configuration and velocities at one instant, or the configurations at two instants, render the configuration at any other earlier or later instant theoretically calculable. That is to say, the configuration at any instant is a function of that instant and the configurations at two given instants. This statement holds throughout physics, and not only in the special case of gravitation. But there is nothing that could be properly called 'cause' and nothing that could be properly called 'effect' in such a system.

Russell's position depends on distinguishing between different types of scientific law. Causal laws are those of the form: 'A causes B'. However there are other types of scientific law. Russell mentions functional laws relating variables, and laws expressed by differential equations. He could have added probabilistic or statistical laws, such as the law that radioactive emissions follow a Poisson distribution. Russell's thesis is that causal laws are useful only in daily life and in the infancy of a science, and are not to be found in an advanced science.

I believed this thesis for many years, and I still think it is correct for theoretical physics. However Jon Williamson persuaded me that it cannot be true for all advanced sciences, since it is plainly false for medicine. Considerations of causality arise at every step in medicine. For example a doctor who carries out a medical diagnosis is attempting to ascertain the cause of a patient's symptoms. If the patient suffers from pains in the chest, it is most important to know whether these are caused by lung cancer, angina, a bacterial infection of the bronchi, or something else altogether. The treatment given will 
be quite different for different causes. We see from this that the notion of cause and causal laws play a crucial role in medical practice.

The same applies to medical research, as can be illustrated by one of Pasteur's famous medical discoveries ( $c f$. Dupré [1994], pp. 330-40). In June 1879, Pasteur collected pus from the boil of one of his assistants, and discovered that it contained a bacterium which was later named staphylococcus. In February 1880 Pasteur took pus samples from deep in the bone of a little girl aged 12 being operated on for the bone disease osteomyelitis. He discovered that the pus contained a bacterium of the same type. This led him to conclude that boils and osteomyelitis are both caused by the same bacterium. This was a very significant discovery since it showed that a serious disease located deep in the tissues had the same cause as a superficial and generally slight illness. The result eliminated the difference between internal and external pathology. Note that this most impressive discovery was the discovery of a causal law.

Discoveries in medicine are used for either the prevention or the cure of diseases. In the case of staphylococcal infections, preventative measures were relatively easy. It was a matter of preventing the pathogenic bacterium entering the body through greater care about hygiene and antisepsis. The discovery of a cure proved much harder. It required finding a substance (an antibiotic) which would kill staphylococci in a patient's body without harming the patients. Fleming discovered penicillin in 1928, but it required a lot of development work both by him, and later by the Oxford team of Florey, Chain, and others before penicillin could become an effective antibiotic. (For details see Macfarlane [1984], especially pp. 185-6.) In fact it was not until May 1941 that the first patient was cured of a staphylococcal infection (a 4 inch carbuncle on the back) using penicillin. Hence 62 years elapsed between the discovery of the cause of a group of illnesses, and the development of a successful cure. However it is worth noting that the discovery of the cause was a precondition for developing a cure.

Returning now to Russell, we can see that medicine completely refutes his claim that causal laws belong only to the infancy of science. Medicine has been highly successful and produced cures which would have been regarded as miraculous in a former age. Thus medicine has every claim to be an advanced science, and yet makes essential use of the notion of cause and of causal laws. Russell, however, was correct in his claim that non-causal mathematical laws have replaced causal laws in theoretical physics. His mistake was to assume that the same applied to every advanced science. This is an instance of a saying of Wittgenstein's ([1953], §593):

A main cause of philosophical disease - a one-sided diet: one nourishes one's thinking with only one kind of example.

It also shows that one must study history and philosophy of science, and not philosophy of science without the history. Only the history of science can provide the variety of examples needed for philosophy of science.

To sum up then: Russell's thesis appears to be true of some advanced sciences, e.g. theoretical physics, but false of others, e.g. medicine. This situation poses the following question. Why is it that some advanced sciences can dispense with the use of causal laws, whereas such laws continue to play a central role in other advanced sciences? As we shall see later, it is a point in favour of the action-related theory of causality that it can provide a satisfactory answer to this question. Let us now turn to a giving a preliminary exposition of this theory. 


\section{Preliminary Exposition of the Action-Related Theory}

The basic idea behind the action-related theory of causality is quite simple. It is that causal laws are useful and appropriate in situations where there is a close link between the law and action based on the law. The concept of cause has evolved in such a way that the transition between a causal law and carrying out actions based on that law is particularly easy and simple. It must be stressed, however, that the actions to which causal laws are related are of two forms, which we will describe as (i) productive, and (ii) avoidance. As we shall see, the introduction of avoidance actions is one of the main points where the present action-related theory differs from the agency theory of Menzies and Price.

Let us take productive actions first. These arise when our law is that A causes B. Someone wants to produce the effect B and does so by instantiating the cause A. Suppose for example that a member of the Praetorian Guard wants to kill the Roman emperor. He knows the causal law that a dagger in the heart causes death. So on the next occasion that the emperor is inspecting his troops, he rushes forward and stabs him in the heart, thereby achieving his goal.

Causal laws can, however, also be connected to an avoidance action. This arises when the law is that A causes B, but now someone wants to avoid the effect B or prevent it from occurring. One strategy which such a person might adopt would be to ensure that A does not occur. Naturally this is not a completely fail-safe method of avoiding B, since there might be another cause A* say of B which produces B even though A does not occur. Nonetheless such avoidance strategies based on causal laws are often effective.

Another type of avoidance strategy is based on pre-empting A from producing its normal effect B. Generally we say that A causes B when A produces B under normal circumstances, or ceteris paribus. Pre-emption of A's causing B is said to occur if we alter some of these ceteris paribus conditions to ensure that A does not have its usual effect. Our earlier dagger example can be extended to give an instance of an avoidance strategy based on pre-emption. It was in fact quite common in late $16^{\text {th }}$ and early $17^{\text {th }}$ century Europe for those in powerful positions to be stabbed by fanatical assassins. To avoid such a fate, James VI of Scotland and I of England used to wear padded clothing. King James knew well the law that, ceteris paribus, daggers plunged into someone cause death or painful injury, and he was taking an avoidance action based on attempting to pre-empt the well-known effects of stabbings with a dagger.

Avoidance actions are of great importance in medicine. Thus, to take the Pasteur example of the previous section, if we want to avoid having boils, we must take care to avoid being infected by staphylococci. If we have a boil, we should seek to eliminate it by taking an antibiotic which kills staphylococci.

The action-related theory of causality explains the value of causal laws both in everyday life and in medicine. Everyday life imposes on us the constant necessity for taking action, and so it is obviously convenient to cast our common-sense knowledge in a form which is closely linked to action, that is to say in the form of causal laws. In medicine too a doctor has to try to cure his or her patients, and so is under an obligation to act. Thus medical knowledge, even though it involves advanced scientific considerations going far beyond common sense, must still be cast in a form which is closely linked to action, that is to say in the form of causal laws.

Turning now to the laws of theoretical physics, I do not of course want to deny that these too are linked to practical applications and actions. Thus, Maxwell's equations 
can be used for making radio transmitters, or quantum mechanics for making nuclear weapons. However the connection between the laws and the corresponding practical actions is much more remote in such cases than it is in everyday life or medicine. In the examples from physics, long mathematical calculations are needed, approximations must be made, and, usually, additional empirical assumptions introduced, before a theoretical scheme such as Maxwell's equations can be connected to a practical problem such as radio transmission. As the link between theoretical knowledge and practical action is here not at all close, laws other than causal laws can make their appearance, and indeed prove more convenient than causal laws. This constitutes a partial justification for Russell's claim that as a science advances it abandons the use of causal laws, since this claim is in fact true of theoretical physics, even though it is false of medicine.

The action-related theory of causality has, however, something more to say about the case of physics. Suppose we start with very theoretical laws of physics such as Maxwell's equations or Schrödinger's equation, and then through mathematical calculations, the construction of more detailed models, etc. gradually move towards some practical application. The action-related theory of causality suggests that, as we approach practical actions, the causal laws which were absent at the very theoretical level might well reappear. This does indeed seem to be the case as is illustrated by the following simple example.

Let us consider the ideal gas law $\mathrm{PV}=\mathrm{RT}$. This is a law of functional form relating the three variables $\mathrm{P}=$ pressure, $\mathrm{V}=$ volume, and $\mathrm{T}$ temperature. The law is clearly not of causal form since we cannot say that any one of the variables, e.g. V, is a cause, and another, e.g. P, is an effect. The variables are all on a par. If, however, we apply the law to a particular concrete situation where a gas is being manipulated in some way, causal notions do reappear. However what counts as a cause may differ in different applications.

The law as we know holds approximately for some gases under certain conditions. Suppose we are handling such a gas under these conditions. Let us first suppose (see figure 1) that this gas is in a cylinder one end of which is closed by a piston which can be moved in or out.

\section{Figure 1}

The apparatus is held at constant temperature. It follows from the ideal gas law that if the piston is moved in, the pressure of the gas increases; whereas if it is moved out, the gas's pressure decreases. But now we can consider the change in volume as the cause and the change in pressure as the effect. This is because, by means of the piston, we can manipulate the volume in order to produce changes in the pressure. Since there is now a close positive connection between the law as applied in this specific case, and the corresponding action, causality has reappeared just as the action-related theory would suggest that it should.

Let us contrast this with a different application of the ideal gas law. Suppose our gas is now in a sealed container made of such a material that it can be regarded as having constant volume for the range of temperatures which occur. Suppose this sealed container can be heated by a bunsen flame as shown in figure 2 , or cooled by being placed in a refrigerator. 


\section{Figure 2}

It follows from the ideal gas law that if the temperature increases, the pressure of the gas will increase; whereas if the temperature is decreased, the gas's pressure will decrease. Once again causality has reappeared in a specific case, but now it is the change in temperature rather than the change in volume which acts as the cause, since manipulating the temperature produces changes in the pressure.

This simple example suggests a reason why, in the development of theoretical physics, it may have proved advantageous to replace causal laws with laws of other kinds. A single functional law such as the ideal gas law yields different causal laws when applied in different situations. This functional law thus in a sense summarises a number of different causal laws and thus produces greater economy than could have been achieved by the exclusive use of causal laws. The advantage of non-causal laws may therefore be that they allow greater economy; their disadvantage that they create a greater gap between the law and action based on the law. This is a gap which may need to be filled by mathematical calculations, approximations, the construction of models, and the other devices mentioned above.

\section{Differences between the Action-Related Theory, and the Agency Theory of Menzies and Price}

As already remarked, the action-related theory was developed from the agency theory expounded by Menzies and Price in a series of important papers (Price [1991], [1992] and Menzies and Price [1993]). It is time now to compare the two theories explaining their differences and the points they have in common. In this section I will discuss three points on which the two theories differ, while in the next section I will deal with the important aspect on which they agree, namely the explanation of causal asymmetry.

In his [1991], Price devotes some space to arguing in favour of evidential decision theory and against causal decision theory. He does so by considering the following medical example. It has long been recognized that in many people susceptible to migraine, an attack tends to follow the consumption of chocolate. It thus seemed that, as least for this class of people, eating chocolate caused migraine, so that to avoid migraine they would be well-advised to give up chocolate. Recently, however, a new theory has been devised according to which eating chocolate is not after all a cause of migraine. Migraine is brought about by a physiological state, and this pre-migrainous state (or PMS) increases the desire for chocolate, thus producing the observed correlation between eating chocolate and the subsequent onset of a migraine attack.

Let us suppose that this new theory is true. There is then no point in a migraine sufferer refraining from eating chocolate, since this will have no influence on whether a migraine attack occurs or not. At any rate this seems to be the conclusion of a causal decision theory, i.e. one which takes causes into account when making decisions. If, however, our migraine sufferers rely on an evidential decision theory, i.e. one which bases decisions on the statistical evidence obtained directly from the data, then it would seem that they should continue to refrain from chocolate, since there is still a statistical correlation between eating chocolate and the onset of a migraine attack. In this case, then, causal decision theory and evidential decision theory give different results, and, as 
the recommendation of causal decision theory seems intuitively correct, this is an argument in its favour and against evidential decision theory.

Despite this difficulty, Price attempts to defend evidential decision theory against causal decision theory, but I have to confess that I cannot see the point of so doing. I would favour not just a causal decision theory, but what could be called a maximal decision theory in which any established scientific law of whatsoever type (causal or noncausal) is taken into account in making a decision. Indeed to ignore any established piece of scientific knowledge in making a decision would seem to be foolish. So, for example, a group of scientific experts when deciding whether to fund a project for building a quantum computer might carry out elaborate calculations based on the laws of quantum mechanics in order to judge whether such a machine was really feasible. No one would say surely that they were wrong to take quantum mechanics into account. They would rather be wrong to ignore quantum mechanics. In the same way a doctor would surely be wrong to ignore established causal laws regarding the onset of a migraine attack when giving dietary advice about how to control the condition. Interestingly Menzies and Price in their joint paper state that Menzies, unlike Price, does support the need for causal decision theory ([1993], p. 190).

Price's support for evidential as opposed to causal decision theory may arise from a generally empiricist rather than realist outlook. At all events, he seems to be sympathetic to the kind of neo-empiricist, neo-Humean probable sequence analysis of causality which, as we saw, was adopted by Russell. It is not that Price himself adopts such an analysis. It is rather that he suggests (and this is a suggestion not a definite claim) that some kind of neo-Humean probable sequence analysis of causality may be viable if we add to it the notion of agency. Thus he says ([1991], p. 158):

... my suggestion is ... that in order to rescue the promised rewards of the probabilistic approach to causality, we should avail ourselves of the idea, often felt to be independently appealing, that the notion of agency is crucial to an understanding of causality.

The following passage makes a similar point and brings out its relation to Price's critique of causal decision theory ([1991], p. 173):

This means not only that there is no need for a distinctively causal decision theory, but also that we may characterize causal regularities as associative regularities that continue to hold from the free agent's distinctive point of view.

In contrast to Price, I favour a realist rather than empiricist point of view, and this leads naturally to a different kind of philosophical theory of causality. An analogy with probability should make this clear. Suppose we are trying to give a philosophical account of probability as it appears in scientific theories. Those who favour an empiricist point of view will be led naturally to the frequency theory of probability which gives an empiricist/operationalist definition of probability in terms of observable frequency. Those who are more inclined to realism will prefer a propensity theory of probability in which probability is an undefined concept, characterised by a set of axioms, and connected only indirectly with observation.

The situation is exactly the same regarding philosophical accounts of causality. Those like Price who have an empiricist outlook will favour trying to define cause in terms of sequences of observable events, together with, in his case, the notion of agency, 
since, as he says ([1991], p. 173): 'Agency is something of which we all have direct experience.' Those of a realist persuasion, however, will not attempt to define cause in terms of what can be directly experienced. Cause will rather be regarded as an undefined sui generis notion, which is connected only indirectly with observation.

I now come to the last, and perhaps key, difference between the agency theory and the action-related theory. To see what this is, let us consider two general accounts of the agency theory of causality. The first comes from Price ([1992]) where he is considering 'the agency or manipulability theory of causation in general', and writes ( $p$. 514):

Roughly, to think of A as a cause of B is to think of A as a potential means for achieving (or making more likely) $\mathrm{B}$ as an end.

The second comes from Menzies and Price ([1993], p. 189):

... the common idea to agency accounts of causation is that an event A is a cause of a distinct event $\mathrm{B}$ just in case bringing about the occurrence of $\mathrm{A}$ would be an effective means by which a free agent could bring about the occurrence of B.

These characterisations of agency theories of causality only mention what we have called productive actions based on a causal law, and not avoidance actions based on such a law. Yet avoidance actions are very important, particularly in medicine. It is true that smoking causes lung cancer, but this does not mean that smoking is an effective means by which a free agent could give himself or herself lung cancer. It is rather that refraining from smoking is a sensible strategy for trying to avoid getting lung cancer. The term 'agency' does indeed suggest the productive action, and this is why I have introduced the term 'action-related' which suggests that it is possible to base different types of action (both productive and avoidance) on causal laws. At all events a consideration of avoidance actions will, as we shall see in section 6, be most important for answering some objections to the action-related theory of causation.

\section{Explanation of Causal Asymmetry}

Having dealt with the differences between the agency theory and the action-related theory, I will now turn to the important feature they have in common, namely the explanation of causal asymmetry. I will begin by expounding Price's views on this question as given in his [1992], but my aim this time is not to criticise these views (with which I agree), but rather to add some additional points in their favour.

Price begins his paper as follows ([1992], p. 501):

Here are two features of causation that an adequate theory of causation might be expected to explain: (i) the causal relation is asymmetric - if A is a cause of B then $\mathrm{B}$ is not a cause of A; (ii) effects never (or almost never) occur before their causes.

Price goes on to criticise Hume's treatment of this matter. According to Hume, A causes $\mathrm{B}$ if $\mathrm{A}$ and $\mathrm{B}$ are constantly conjoined and A occurs before B. However Price objects that this makes the causal and temporal arrows related by definition instead of offering an 
explanation of this relation. Moreover the definition rules out backward causation which Price holds to be logically possible and perhaps even to occur in some cases. In contrast to Hume's account, the agency theory of causation, according to Price, does really offer a very satisfactory explanation of causal asymmetry. As he says ([1992], p. 515):

... the agency account of causation ... has a significant and largely unrecognized advantage: it is particularly well placed to explain the nature of causal asymmetry, and its prevailing orientation in time. For it is able to say that the asymmetry of causation simply reflects (or better, perhaps, projects) that of the means-end relation. Causes are potential means, on this view, and effects their potential ends. The origins of causal asymmetry thus lie in our experience of doing one thing to achieve another; in the fact that in the circumstances in which this is possible, we cannot reverse the order of things, bringing about the second state of affairs in order to achieve the first. This gives us the causal arrow, the characteristic alignment of which with the temporal arrow then follows from the fact that it is normally impossible to achieve an earlier end by bringing about a later means.

To this passage Price appends an interesting footnote (16) about backward causation ([1992], p. 515):

The status of backward causation then turns on the question as to whether there are or could be exceptions: cases in which we could coherently act for past ends. Dummett (1964) has defended the conceptual possibility. A number of writers (including myself, in my 1984) have suggested that quantum mechanics might provide some actual examples.

Although backward causation is an intriguing possibility, we can be certain that it almost never occurs in practice, and so I will ignore it in the rest of the paper. I will therefore assume the following principle:

Human action cannot change the past.

From (*) and the action-related theory of causality, a second principle follows immediately, namely:

'A causes B' cannot hold, if B occurs earlier in time than A.

According to the action-related theory, if 'A causes B' holds, then we can either produce or avoid B by manipulating A. If, however, B occurred earlier in time than A, such manipulations would involve altering the past, which, by $\left(^{*}\right)$ is not possible. Hence $(* *)$ is true.

From $(* *)$ it follows in turn that if A causes B, then either B occurs later than A, or $\mathrm{A}$ and $\mathrm{B}$ are simultaneous. Let us consider these two cases in turn. If $\mathrm{B}$ is later than $\mathrm{A}$, then it is immediate by $(* *)$ that B cannot cause A. So the asymmetry of causation is established in this case. The case in which A and B are simultaneous is more complicated, and requires more careful consideration. 
Kant mentions simultaneous causation, although it raises some difficulties for his views. He writes ([1781/7], A202/B248):

At this point a difficulty arises with which we must at once deal. The principle of the causal connection among appearances is limited in our formula to their serial succession, whereas it applies also to their coexistence, when cause and effect are simultaneous. For instance, a room is warm while the outer air is cool. I look around for the cause, and find a heated stove. Now the stove, as cause, is simultaneous with its effect, the heat of the room. Here there is no serial succession in time between cause and effect. They are simultaneous ...

Simultaneous causation also raises a problem for Hume's account of causation in addition to those mentioned by Price which we described earlier.

Medicine affords many examples of simultaneous causation. A tumour may be the cause of a patient's pain, although tumour and pain occur simultaneously. In such cases of simultaneous causation, we are no longer dealing with a situation in which one event causes another event, but with a situation in which two processes are involved, one of which causes the other. Thus one process (the burning of fuel in the stove) causes another process (the temperature of the room remaining above that of the outside air). In the medical case, we can suppose that the tumour and pain begin and develop together. The more the tumour grows, the worse the pain. Although the causal relation now holds between processes which occur simultaneously, the asymmetry of causation still applies. The stove causes the elevated temperature of the room, but not vice versa. The tumour causes the pain, but the pain does not cause the tumour. Once again the action-related theory of causation explains this asymmetry. If we want to heat the room, we can light the stove, but we cannot ensure that the stove is burning by altering the temperature of the room, assuming that we have only $18^{\text {th }}$ century technology at our disposal, and there are no thermostats and heating control systems. Similarly it may be possible to eliminate the patient's pain by manipulating the tumour, for example by excising it surgically. However it is clearly not possible to alter the tumour by acting upon the pain in some way.

Thus the agency theory, and its generalisation the action-related theory of causation provide a simple and fully satisfactory explanation of causal asymmetry. This is a very strong point, indeed perhaps the strongest point, in favour of these theories for no alternative view of causation seems to give an adequate explanation of causal asymmetry. The case of simultaneous causation is particularly difficult to deal with on any other account. There are, however, quite a number of objections to the action-related theory of causation. I will consider and try to answer some of these objections in the next section of this paper.

\section{Objections to the Action-Related Theory}

The main objection to the action-related theory of causality concerns causal laws of the form A causes B, where the cause A cannot be either instantiated or prevented from occurring by human action, but, so to speak, arises spontaneously in the course of nature. In such cases we can neither produce the effect $B$ by deliberately instantiating the cause A, nor prevent the effect B by preventing the cause A, from occurring. It therefore looks as if the link between causal laws and actions based on them which is postulated by the 
action-related theory is broken, and that therefore the action-related theory fails to apply in such cases. This objection is made by Woodward as follows ([1999], pp.209-10):

Another criticism lodged against traditional manipulability theories is that ... they face great difficulties in explicating causal claims (e.g., 'changes in the position of the moon cause changes in the tides on earth') for which the relevant human manipulations are impossible.

I will return to this example of Woodward's later on, but will begin by illustrating the problem with the simple case of investigating possible causes of the lawn being wet. Two possible cases are the following.

The sprinkler caused the lawn to become wet

Rain caused the lawn to become wet.

Both seem to be perfectly legitimate instances of causation. Yet while (1) fits the actionrelated theory perfectly, (2) does not appear to do so at all. We can turn on the sprinkler whenever we wish the lawn to become wet, and so base our action on a causal law linking the functioning of the sprinkler with the wetness of the lawn. However we cannot turn the rain on or off, and so the link between a causal law connecting rain with the wetness of the lawn and corresponding actions seems to be broken.

What can be done about such cases of unmanipulable causes? In fact there are two strategies for dealing with them. The first depends on using avoidance actions. Some such actions are based on pre-emption. As we remarked earlier, most causal laws of the form 'A causes B' should really be written 'A causes B under normal circumstances'. Even if we cannot prevent the cause A from occurring, we can often alter the circumstances in order to pre-empt the cause A from having its usual effect B. This is an avoidance action based on the causal law. In other cases, there is no way of pre-empting the cause A from producing B, but, even if B does occur, we can still avoid it. This is another sort of avoidance action based on the causal law. The second strategy for dealing with unmanipulable causes is to use Russell's suggestion of eliminating causal laws in favour of other sorts of law such as functional laws. This strategy works well for examples from physics and astronomy, where, as already remarked, Russell's claim that causality can be eliminated is largely correct. I will now illustrate these various strategies by considering a few examples.

Let us start with the case of rain causing the grass to become wet. Here it is a simple matter to pre-empt the cause so that it does not have its usual effect. Thus when rain stops play in a cricket match, the groundsmen bring out the covers, so that the wicket remains dry, thus ensuring that play can be resumed as soon as the rain stops. The earlier example of James I wearing padded suits to prevent the usual effects of dagger blows is similar.

Unmanipulable causes cannot, however, always be pre-empted to prevent their usual effects. For example earthquakes are caused by friction between continental plates, but this friction on the boundaries of continental plates cannot be pre-empted in any way to prevent earthquakes. This causal law can, nonetheless, be used as the basis of avoidance action. To take the simplest such action, those who are particularly keen to avoid earthquakes can refrain from going into areas on the boundary between continental plates. 
This strategy for dealing with unmanipulable causes depends on consideration of avoidance actions. Now earlier I criticised the agency theory of Menzies and Price on the grounds that it considered only productive actions and not avoidance actions. This criticism can now be reinforced by remarking that this restriction prevents them from giving a satisfactory account of unmanipulable causes. Thus they deal with the earthquake case by saying that the causal law enables seismologists to simulate earthquakes by ([1993], p. 198) 'their artificial simulations of the movement of continental plates.' This does not seem to me a satisfactory solution to the problem. The whole point of an agency or action-related theory of causality is to link causal laws with practical human actions in the real world. Simulations are better considered as a theoretical preliminary to practical action rather than as part of such action.

A consideration of avoidance actions also helps to deal with another class of unmanipulable causes, namely those giving the causes of events which happened in the past. Consider the following example.

The collision of the Earth with an asteroid or large meteor caused the extinction of the dinosaurs.

There is now a great deal of evidence for the historical statement $\mathrm{H}$, and it is widely believed by palaeontologists. Yet we cannot go back in time and prevent the collision of the Earth with the asteroid or large meteor, or alter its effect - the extinction of the dinosaurs. These events occurred in the past and cannot now be changed. How then can the causal statement $\mathrm{H}$ be related to human actions?

My answer regarding this and similar examples is that historical statements involving causality such as $\mathrm{H}$ should be taken as particular instances of more general causal laws which could apply today as well as in the past. Regarding our example $\mathrm{H}$, the general causal law would be something like the following.

The collision of the Earth with an asteroid or large meteor would cause the extinction of a large percentage of the then living species.

In fact many of the scientific arguments which established $\mathrm{H}$ also established $\mathrm{T}$ because they exhibited mechanisms which linked the collision with the extinction of a large percentage of the living species of the time. Now $\mathrm{T}$ is a law on which human action can certainly be based. Indeed there is currently a project to keep a careful watch on the orbits of asteroids and large meteors to see if there is a risk of any such object colliding with the Earth. If there were such a risk, the object could be broken up or deflected from its course by a suitable use of missiles, and so the human race might escape the fate of the dinosaurs. My claim here is a particular instance of the maxim that the past can sometimes be a guide to the present. The causes of a historical event might still operate in the present and might therefore form the basis of human action, even though the past cannot be changed.

I now turn to the second strategy for dealing with unmanipulable causes, namely Russell's suggestion of eliminating causal laws in favour of other sorts of law such as functional laws. An example for which this treatment seems appropriate is the following: 'The change of seasons is caused by the movement of the Earth in its orbit round the Sun.' This apparently causal law can easily be replaced by a functional law. The seasons 
depend on the angle of elevation ( $\theta$ say) of the sun at mid-day, and on the direction of change $d \theta / d t$ of this angle. A relatively simple functional law relates $\theta, d \theta / d t$ to the position $\varphi$ say of the Earth in its orbit round the Sun. An example like 'the background radiation in the universe was caused by the big bang' can be dealt with in the same way, although the non-causal laws involved will obviously be more complicated. Woodward's example, quoted earlier, of changes in the position of the moon causing changes in the tides on earth could also be dealt with by this Russellian technique.

It might still be objected that this way of dealing with counter-examples is hardly satisfactory since the laws claimed to be non-causal are perfectly causal according to the standard concept of cause. However, this objection does not take into account the fact that the action-related theory of causality, like any other philosophical theory, provides an explication, in the sense of Carnap, of the concept with which it deals. According to Carnap we start philosophising by considering a pre-formal concept or explicandum, and the aim is to replace this with an improved concept, the explicans. Now it is indeed required that the explicans and explicandum overlap to a large extent, but still, because the explicans is a replacement, it is perfectly legitimate that there should be a few instances of the explicans which are not instances of the explicandum, and a few instances of the explicandum which are not instances of the explicans. The standard example of the latter case being the scientific explication of the concept of fish which results in the whale ceasing to be a fish and becoming a mammal. (This is what Lakatos called monster-barring.)

As we saw, Russell's neo-Humean explication of cause made it correct to say that night causes day, whereas according to the ordinary notion of cause, day follows night, but it is not caused by night. The action-related theory of causality agrees with the ordinary notion of cause in this instance, but disagrees in the related example of night and day being caused by the rotation of the Earth. According to the action-related theory, this latter example should no longer be considered as a genuine example of a causal law, and should be replaced by a functional law, as of course can easily be done.

Thus the action-related theory of causality, being an explication, does to some extent alter the boundaries of the concept of cause. Yet this alteration is in fact very small. Nearly all standard uses of the concept of cause are closely related to actions of a productive or avoidance kind in exactly the way that the action-related theory prescribes.

I turn lastly to a more general objection to the action-related theory of causality. It could be claimed that this theory is unacceptably anthropocentric. In particular it looks as if the theory leads to the absurd conclusion that if there were no humans, and so no human actions, there would be no causes. Yet surely friction between continental plates would continue to cause earthquakes even if there were no humans, and no doubt many dinosaurs suffered from pain caused by tumours.

My answer to this difficulty falls into two parts. I will first argue that the actionrelated theory does not prevent causal laws being objective and potentially human independent. However I will then qualify this by arguing that the action-related theory does give the concept of cause an anthropocentric aspect, and that this is desirable rather than harmful.

The first line of argument runs as follows. The natural world is to be considered as highly complicated and subject to many variations, while at the same time obeying quite a number of laws and regularities. From this profusion of laws, humans, naturally enough, pick out those which are useful to them in carrying out actions either to achieve desirable goals or to avoid undesirable situations. This is why there is, in particular, a 
search for laws which are of causal form, and so can be related to human action in a simple and straightforward way. These laws have the property of being closely linked to human action, but they hold objectively quite apart from this property. Indeed if they did not hold objectively, they would not be useful as a basis of action. This is why we can suppose that so many causal laws would continue to operate in the absence of humans.

The point could be put in this way. The fact that a causal law is a law of nature means that it holds objectively independently of humans. Yet the fact that it has been picked out from the complexity of the natural world by humans as a causal law shows that it is closely related to human action. This is why causal laws can at the same time be objective and potentially human independent, while having an anthropocentric aspect.

\section{Extension of the Theory to the Indeterminate Case}

Most of the examples of causal laws considered so far in this paper have used what could be called determinate causality, which can be explained as follows. A causes B involves a determinate notion of causality if, ceteris paribus, the instantiation of $\mathrm{A}$ is always followed by B. In the eighteenth and nineteenth centuries, causes were considered to be determinate. A dagger in the heart causes death, because death always follows such a dagger blow. Now, however, we are quite happy to say that smoking causes lung cancer, although the habit of smoking does not always result in lung cancer. In fact less than 5\% of smokers get lung cancer. Thus we are dealing here with what could be called indeterminate causality.

It is rather difficult to say when this notion of indeterminate causality arose.

Kant, for example, writing at the end of the eighteenth century, regards causes as completely determinate, as is shown in the following passages from the Critique of Pure Reason ([1781/7], A144/B183):

The schema of cause, and of the causality of a thing in general, is the real upon which, whenever posited, something else always follows.

$([1787], \mathrm{B} 5)$ :

... the very concept of a cause ... manifestly contains the concept of a necessity of connection with an effect and of the strict universality of the rule, ...

Russell in his [1913] paper does say that causality is probabilistic rather than necessary, but he clearly regards this as something of an innovatory view. Moreover his example (striking a match causes it to ignite) is not really an example of indeterminate causation. The law should be understood in the sense that striking a match causes it to ignite under normal circumstances. These normal circumstances are never of course exactly specified, but they are meant to exclude cases such as the match being damp. Although it is true, in the sense of indeterminate causation, that smoking causes lung cancer, we do not want to claim that smoking causes lung cancer under normal circumstances, since a very high percentage of perfectly normal smokers do not get lung cancer.

My suspicion is that the notion of indeterminate causality actually arose with the development of medical epidemiology, so that familiar examples such as smoking causes lung cancer are in fact among the first examples of indeterminate causality in modern times. However this is an historical conjecture which would need further investigation. 
Developments in quantum mechanics may also have influenced the emergence of the concept of indeterminate causality in the twentieth century.

As the action-related theory of causality expounded in this paper has been based largely on examples of determinate causality, the question naturally arises as to whether it can be extended to indeterminate causality. Indeterminate causality is more complicated than determinate causality. It involves networks of causes, and it also involves rather complicated relations between causality and probability. Nonetheless I would say that the tools do exist for extending the action-related theory to indeterminate causality. They consist principally of two notions developed by Pearl (see his [1988] and [2000]), namely (i) Bayesian networks and (ii) the $d o$-calculus. However, this rather technical extension of the theory would require a paper on its own, and so cannot be dealt with here.

\section{Acknowledgements}

I would like to thank the AHRB for providing a term of sabbatical leave in the spring of 2003 during which I was able to work on this paper. Earlier versions of this paper were read at the Philosophy Department's seminar in King's College London, and at Birkbeck Philosophy Society. I would like to thank those who offered many helpful comments on those occasions. Drafts of the paper were read by Raffaella Campaner, David Corfield, Ladislav Kvasz, David Papineau, Jon Williamson, and two anonymous referees. I am grateful for their comments and criticisms which have helped to improve the paper.

Department of Science and Technology Studies

University College London

Gower Street

London WC1E 6BT

U.K.

donald.gillies@ucl.ac.uk

\section{References}

Bacon, F. [1620]: Novum Organum. English translation in Ellis. R.L. and Spedding, J. (eds.), The Philosophical Works of Francis Bacon, London: Routledge, 1905, pp. 212387.

Dummett, M. [1964]: 'Bringing about the Past', reprinted in Truth and Other Engimas, London: Duckworth, 1978, pp. 333-50.

Dupré, P. [1994]: Louis Pasteur. English Translation, Baltimore \& London: John Hopkins University Press, 1998.

Kant, I. [1781/7]: Critique of Pure Reason. English Translation by Norman Kemp Smith, London: Macmillan, 1958. 
Macfarlane, G. [1984]: Alexander Fleming. The Man and the Myth. London: Chatto \& Windus.

Menzies, P. and Price, H. [1993]: 'Causation as a Secondary Quality', British Journal for the Philosophy of Science, 44, pp. 187-204.

Pearl, J. [1988]: Probabilistic Reasoning in Intelligent Systems. Networks of Plausible Inference. San Mateo, California: Morgan Kaufmann.

Pearl J. [2000]: Causality. Models, Reasoning, and Inference. Cambridge: Cambridge University Press.

Price, H. [1984]: 'The Philosophy and Physics of Affecting the Past', Synthese, 16, pp. 299-323.

Price, H. [1991]: 'Agency and Probabilistic Causality', British Journal for the Philosophy of Science, 42, pp. 157-76.

Price, H. [1992]: 'Agency and Causal Asymmetry', Mind, 101, pp. 501-20.

Russell, B. [1913] 'On the Notion of Cause', reprinted in Mysticism and Logic, London and New York: Routledge, 1994, pp. 173-99.

Wittgenstein, L. [1953]: Philosophical Investigations. $2^{\text {nd }}$ Edition of English Translation by Elizabeth Anscombe, Oxford: Blackwell, 1963.

Woodward, J. [1999]: 'Causal Interpretation in Systems of Equations', Synthese, 121, pp. 199-247. 
Figure 1

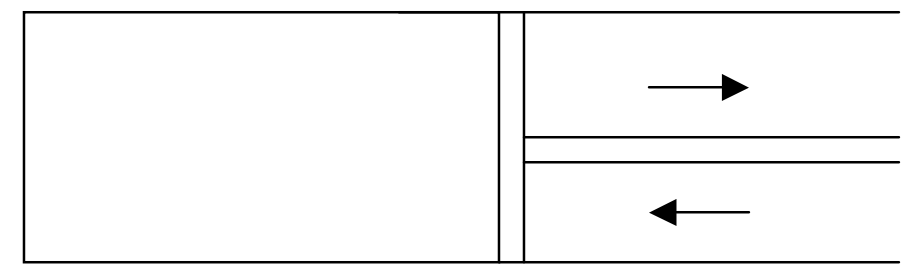


Figure 2

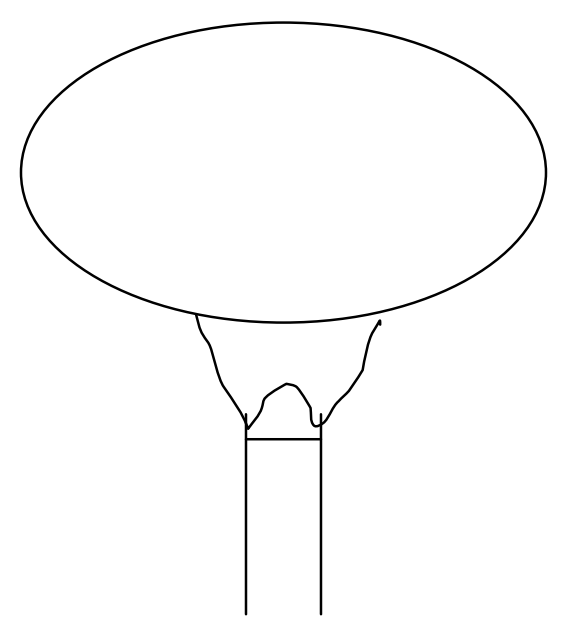

